\title{
Hybridization of Bat and Genetic Algorithm to Solve N-Queens Problem
}

\author{
Abbas Fadhile Jasim Al-Gburi ${ }^{1}$, Syibrah Naim ${ }^{2}$, Aziz Nasser Boraik ${ }^{3}$ \\ ${ }^{1,2}$ School of Computer Sciences, Universiti Sains Malaysia (USM), Pulau Pinang, Malaysia \\ ${ }^{3}$ Faculty of Computer Sciences, University of Aden, Aden, Yemen
}

\section{Article Info \\ Article history: \\ Received Aug 22, 2018 \\ Revised Oct 26, 2018 \\ Accepted Nov 13, 2018}

\section{Keywords:}

Bat algorithm

Genetic Algorithm

Microbat

N-Queens problem

Pulse rate

\begin{abstract}
In this paper, a hybrid of Bat-Inspired Algorithm (BA) and Genetic Algorithm (GA) is proposed to solve $\mathrm{N}$-queens problem. The proposed algorithm executes the behavior of microbats with changing pulse rates of emissions and loudness to final all the possible solutions in the initialization and moving phases. This dataset applied two metaheuristic algorithms (BA and GA) and the hybrid to solve $\mathrm{N}$-queens problem by finding all the possible solutions in the instance with the input sizes of area $8 * 8,20 * 20$, $50 * 50,100 * 100$ and $500 * 500$ on a chessboard. To find the optimal solution, consistently, ten run have been set with 100 iterations for all the input sizes. The hybrid algorithm obtained substantially better results than BA and GA because both algorithms were inferior in discovering the optimal solutions than the proposed randomization method. It also has been discovered that BA outperformed GA because it requires a reduced amount of steps in determining the solutions.
\end{abstract}

Copyright () 2018 Institute of Advanced Engineering and Science. All rights reserved.

\section{Corresponding Author:}

Abbas Fadhile Jasim Al-Gburi, School of Computer Sciences, Universiti Sains Malaysia (USM), Pulau Pinang, Malaysia.

Email: abbaspro7@gmail.com

\section{INTRODUCTION}

$\mathrm{N}$-queens problems (NQP) is the one of research fields that attract researchers attention to optimize the games. The main problem is how to position " $N$ non-attacking queens on the $N * N$ area of chessboard". According M. Bezzel and German chess player [3], it was the generalization of the queen problem by placing the 8 non-attacking queens on chessboard. The first published was in 1848. The purposes of NQP on a chessboard are to modulate $\mathrm{N}$-queens by not having the ability to cross in vertical, horizontal and diagonal directions.

In the 19th century, researchers focused on finding a suitable position to assign queens on a chessboard depending on the game target based on EQP (Eight Queen Problem) as a very interesting problem in this arena $[4,5]$. It is a traditional combinatorial problem, mostly used as a benchmark because of its simplicity and regular structure. Many approaches have been proposed to solve this problem utilized BA, GA and other randomization methods. In [7], the researcher introduced randomization technique that based on randomization in the initialization and moving phases to find the optimal solutions.

The main aim of this hybridization is to develop method between BA and GA that is more sophisticated for the $\mathrm{N}^{*} \mathrm{~N}$ board so that no queen is attacking another queen. This problem able to be solved by the hybridization of BA and GA. Input size for N queens on chessboard is $8 * 8,16 * 16,20 * 20,50 * 50$ and $100 * 100$, the number of possible solutions able to be produced. Figure 1 shows example of position of $\mathrm{N}$ queens based on different size area of chessboard. 

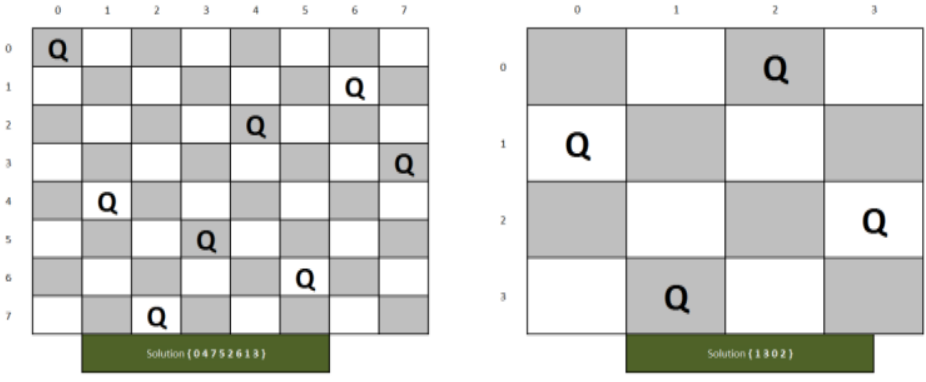

Figure 1. N-Queen solution for board size $8 * 8$ on the left and $4 * 4$ on the right

We formulated the problem, then stated the objective functions to obtain the result, and finally compared with other techniques to obtain the best possible solution. The content of this paper will be from as follows. In Section 2, focuses on the related work similar to this paper. Section 3, definition algorithms of (BA and GA) are introduced. Later in Section 4, the problem formulation and objective function are stated. Section 5 and 6 explains the tuning and experimental design to demonstrate our works, respectively. The result is obtained and explains in the Section 7. Section 8 concludes the paper.

\section{RELATED WORK}

Over the time, there are many authors employ different techniques to solve the $\mathrm{N}$-queens, such as [1] applied genetic algorithms (GA) to solve N-queens problem. In order to improve GA speed and performance, a promising algorithm so-called global parallel genetic algorithm is introduced [1]. The results showed that GA managed to catch multiple solutions for $\mathrm{n}$ th of queens. A research conducted in 2007, [2] showed that the NQP could be effectively solved via heuristic algorithms such as Simulated Annealing (SA) and Tabu Search (TS) by comparing their efficiencies and achievements [7]. In addition, Jordan and Brett [3] considered other board topologies and dimensions as the extensions of the problem to survey known results for the $\mathrm{N}$-queens problem by placing $\mathrm{N}$ queens on an $\mathrm{N}^{*} \mathrm{~N}$ chessboard [7]. For all solution, they give the structure, a framework and references. They also delivered results from the discovery of the intersections of diagonals and investigated number of open investigation areas, stating numerous current researches and new inferences.

In 2004, [4] Ant Colony Optimization (ACO) was achieved to solve (NQP) N-queens problems as 8-Queens. The results show that ACO is able to improve the optimal solution in increasing the speed by improving the computational times for combinatorial optimization problems. In 2010, [5] presents hybridization between different evolutionary algorithms and quantum computing principles such as quantum bits and states superposition. This algorithm is a hybridization between Differential Evolution Algorithms (DEA) and GA [7]. The trial outcomes presented that this combination approach has significant competency and the results improved [7]. In 2012, [6] proposed new method for solving N-queens by using hybridization of Depth First Search (DFS) and Breadth First Search (BFS). The new algorithm suggested assigning the queens on chessboard straight away and the results report the efficiency and runtime of the proposed methodology [7].

Few researchers have hybridized BA in different problems. [8] has implemented the BA with differential evolution strategies. [9] executes a modification for the local search of BA to expand the bats' movement. New operators for the different parts of the solution demonstration have been initialized to diverse the bat population. While [10], presented the planning and scheduling tool for the capital goods industry utilized Discrete Bat Algorithm (DBA) with Krill Herd algorithm (HDBK) to optimize schedules. All these hybridization showed very promising results on standard benchmark functions and significantly improved the original BA.

\section{ALGORITHMS}

\subsection{Bat Algorithm (BA)}

In this section, we describe the bat algorithm, (BA) that based on the echolocation behavior of microbats. Bat algorithm is a bio-inspired algorithm developed by Yang in 2010. BA is a population-based optimization algorithm. This bat algorithm is based on the echolocation behavior of microbats with changing pulse rates of emission and loudness. The bats use a type of bio-sonar echoes to sense prey and evade obstacles to fly in the darkness. The idea of bats behavior; the bats produce a very loud sound and listen for 
the echo that bounces back from the nearby objects. Bats use the time adjournment from the emission and recognition of the echo. From the echoed, the bats can also define the size of the objectives, the distance and even their texture. When hunting for prey, the emission's pulse rate increases.

As indicate, Figure 2 is visibly shows how bat act, by using echolocation to compute distance, and they able to distinguish between food/prey and background barriers. Bats fly arbitrarily with velocity at some position with a frequency $f$ min, wavelength $\lambda$ and loudness A0 to hunt for prey [11]. Bats automatically regulate the wavelength (or frequency) of their emitted pulses and adjust the rate of pulse emission $r \in[0,1]$, relying on the closeness of their target [11]. The loudness can diverge in many ways; however, it can be expected that the loudness vary from a large (positive) A0 to a minimum constant value A min [11].
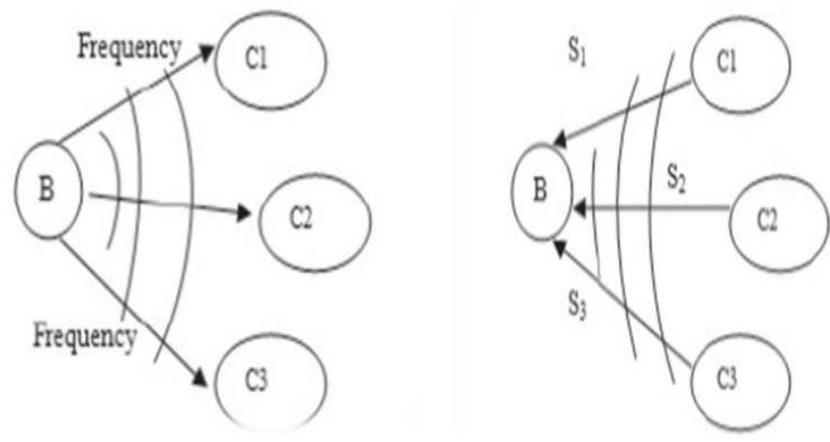

Figure 2. The left side display the bats that send signal with frequency $f$, the right side display how echo signal is used to calculate the distance

\subsection{Genetic Algorithm (GA) to Solve N-Queens Problem}

Genetic algorithm is a population-based metaheuristic method and [1] was originally, considered the first investigation of GA to the N-queen problem. The application of Genetic Algorithm N-queens require non-attacking arrangements such as problem representation, crossover mutation, repair process, selection methods and parameter settings [1]. Furthermore, a simulated evolution algorithm imitate creation of offspring produced from a given population [1]. The population refers to the potential inputs. All expected arrangements of the queens on a chessboard has to define by a collection of many individuals.

Any solutions to the 8 queens' problem can be determined by the assignment of the queens in nonattacking arrangements since every human being can be defined by the presence of appearances [1]. Therefore, each parent having some random arrangement of the queens. Subsequent, every individual regarded as a strong offspring or a weak one. We estimate strength of a human offspring over physical strength, immunity, etc. while in the case of 8 queens, the fitness of a board arrangement can be measured from the number of clashes that occur between the queens so the computation of the fitness of any individual is recognized to number of clashes among attacking positions of queens [1].

\section{PROBLEM FORMULATION}

Three steps of the problem formulation are defined and describe objective function as follows:

a. Initial State: $\mathrm{N}$ queens placed randomly on the board, one per column.

b. Successor function: moving one queen to a new location.

c. Cost: The number of queens that hit each other's.

The objective functions are listed to satisfy two properties:

a. It is maximized at a solution.

b. It is non-negative.

In (1) shows one of many other functions satisfying these two properties, for example (N2) $-\mathrm{L}+\mathrm{C}$ (N2) $-\mathrm{L}+\mathrm{C}$ for every $\mathrm{C} \geq 0 \mathrm{C} \geq 0$. The exact choice is not so important - indeed, they chose $\mathrm{N} 2 / 2 \mathrm{~N} 2 / 2$ whereas they could have chosen $(\mathrm{N} 2)=\mathrm{N}(\mathrm{N}-1) / 2(\mathrm{~N} 2)=\mathrm{N}(\mathrm{N}-1) / 2$.

$$
\mathrm{O}(\text { board })=\mathrm{N}^{2} / 2-\mathrm{L}
$$


For more detailed about the cost penalty of one queen, it is equal to the number of queens that she can check. The fitness of the configuration is equal to the sum of all the queens' penalties separated by two, deleting redundancy counting.

\subsection{Representation N-Queen Problem}

The problem is to place input size queens on according to input size for instants: $8 \times 8,20 \times 20,50 \times 50$, $100 \times 100$ and 500x500 cheeses board so that there is no two queens would attack i.e. there is no two of them are on the same row, column or diagonal. The strategy: For example, we take standard $8 \times 8$ chess, the rows and columns are numbered 1 through 8 . The queens are also numbered 1 through 8 . Since each queen is to be on a different row without loss of generality, we assume queen $i$ is to be placed on row $i$.

\subsection{Hybridization BA and GA}

To solve N-queens using any algorithm techniques, we formulate the problem in term of the objective function. Here is stages of method applied in this study as shown in Figure 3.

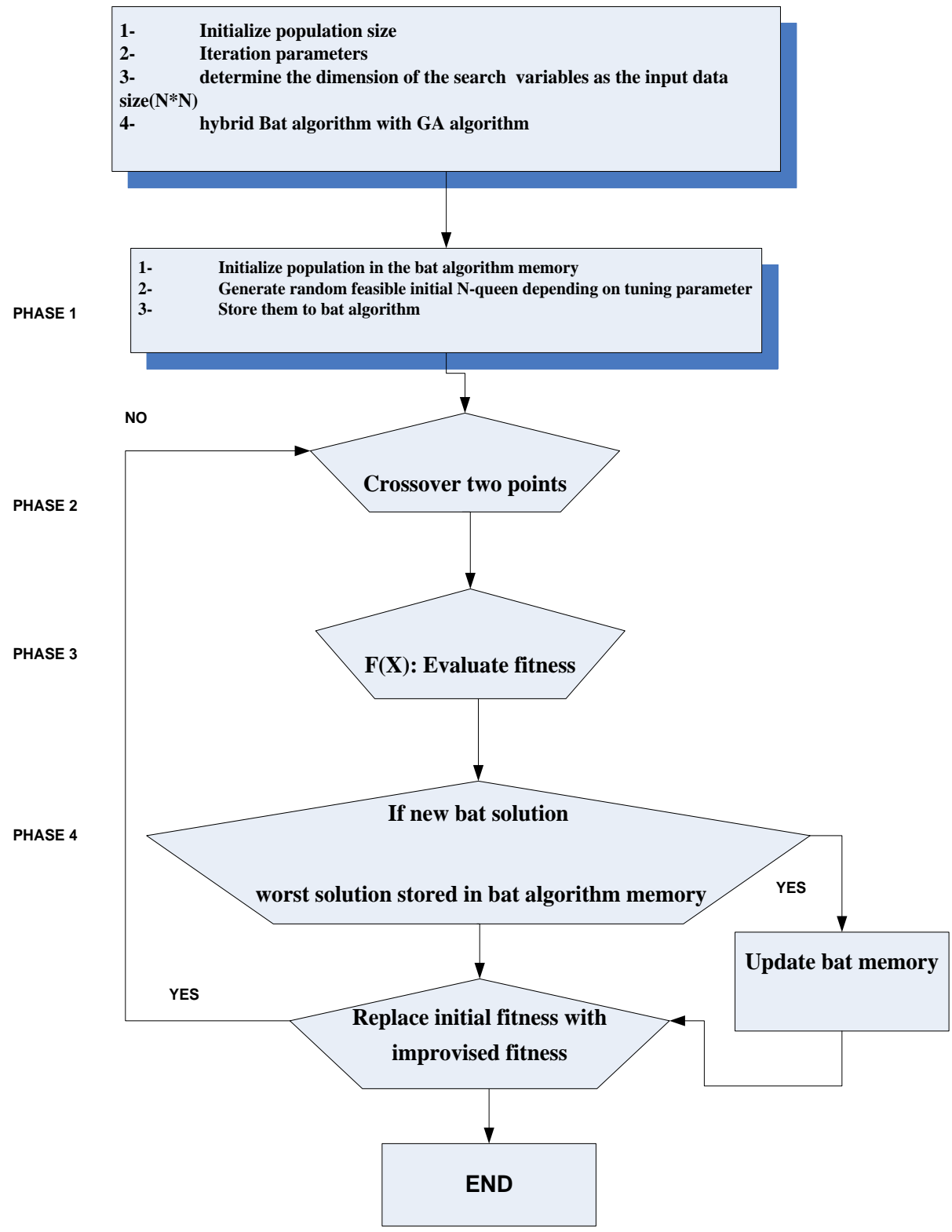

Figure 3. Flowchart of four phases of hybridization between bat algorithm and genetic algorithm 
The first stage includes formulation of the $\mathrm{N}$-queen. The second stage involves adapting bat algorithm to $\mathrm{N}$-queen (i.e., this method is called basic-Bat. Three steps in adapting bat algorithm for $\mathrm{N}$ queen:

a. Initialize population size;

b. Iteration parameters, which mean Loudness (constant or decreasing) and Pulse rate (constant or decreasing). The frequency range determines the scaling. These values need to be changed as necessary which named minimum frequency and maximum frequency;

c. Determining the dimension of the search variables, which consist the number of dimensions and the total number of function evaluations. Finally, the last stages are hybridizing the BA with GA in that we proposed in four phases.

First Phase: -In this phase we apply the initialize population /solutions in order to find best solution when starting number of iterations.

Second Phase: -Presented was chosen a two random points to split parents at this crossover point. More details to the two points crossover operator differs from the one point crossover in the fact that two crossover points are selected randomly:

Parent\#1: 011|101|0101

Parent \#2: $100|111| 0111$

Third Phase: -Evaluate each solution in the population and calculate its fitness value.

Fourth Phase: -Finally in this phase, updates if the solution vector N-Q improves, or not too loud. It was the bast than worst solution was stored in bat algorithm memory, the new best solution as vector Replace initial fitness with improvised fitness.

\section{TUNING THE BAT PARAMETERS}

The tuning parameters of BA may lead to obtain N-Queen solution. The main objective of this experiment is to study the effect of various parameter $r$ (pulse rate) and A (loudness) and to improve the performance when hybridizing BA with GA by selecting value of parameters as the following:

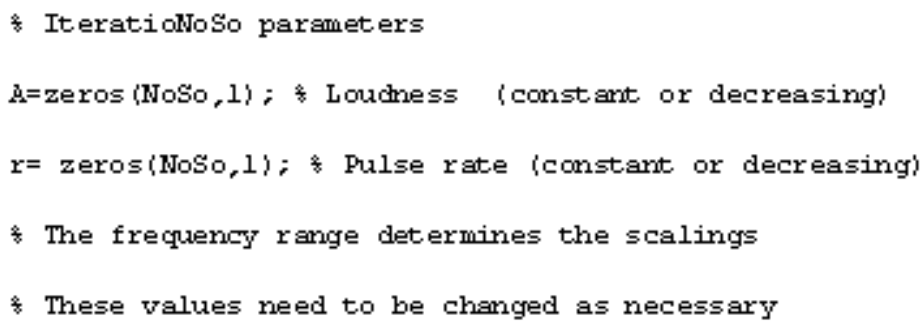

\section{EXPERIMENTAL ANALYSIS}

We evaluate our results on the instances of dataset such as $\mathrm{N}^{*} \mathrm{~N}$ with the same initial configuration. For example, in Bat algorithm different values of $\mathrm{N}$-value will be tested in order to evaluate the new queen and decide the best configuration of the Bat algorithm hybrid with GA. In N-queen, the variables of the new solution are selected based on the current values of the current solution or randomly from possible search space with a probability of $\mathrm{N}$. According to the literature review of the $\mathrm{N}$-queen, the termination criterion is the max number of iteration for each experiment that is predefined before running the system. The experiments were performed on the selected datasets which proposed by input $\mathrm{N}^{*} \mathrm{~N}$ that needed.

The $N * N$ sizes are: $8 * 8,20 * 20,50 * 50,100 * 100$ and 500*500. By following the literatures, number of run was set to ten (ten solutions) for each of the input size and the number of iterations was set to one hundred. The increasing sizes take longer time to execute.

\section{RESULT}

In this section, we present the performances of the proposed hybridized BA-GA algorithm for nqueens with the best results obtained out of 10 runs. The result indicates that the exploration was low and the algorithm focused on $\mathrm{N}$-queen. The next five input size were ran to evaluate the different input values of the $\mathrm{N}$-queen. It will improve the result of the BA. After hybridization between BA and GA the performance of 
the algorithm, reaching high global feasible solution will further improve due to the high exploration rate indicated by $8 * 8$ until $50 * 50$. The best results for all the input size were achieved $8 * 8$ and $20 * 20$. Table 1 stated the result of hybridization of BA and GA. The best results were highlighted as the small penalty acquired (smallest value).

Table 1. Best and Worst Result of the Hybrid BA and GA (BA-GA)

\begin{tabular}{cccccc}
\hline SOL & $8 * 8$ & $20 * 20$ & $50 * 50$ & $100 * 100$ & $500 * 500$ \\
\hline So1 & 0 & 0 & 10 & 42 & 314 \\
So2 & 0 & 0 & 12 & 32 & 332 \\
So3 & 0 & 2 & 8 & 44 & 308 \\
So4 & 0 & 2 & 12 & 34 & 318 \\
So5 & 0 & 2 & 12 & 44 & 302 \\
So6 & 0 & 0 & 10 & 32 & 272 \\
So7 & 0 & 2 & 12 & 28 & 292 \\
So8 & 0 & 0 & 12 & 42 & 314 \\
So9 & 0 & 0 & 12 & 26 & 358 \\
So10 & 0 & 0 & 12 & 36 & 320 \\
Best & $\mathbf{0}$ & $\mathbf{0}$ & $\mathbf{8}$ & $\mathbf{2 6}$ & $\mathbf{2 7 2}$ \\
Worst & $\mathbf{0}$ & $\mathbf{2}$ & $\mathbf{1 2}$ & $\mathbf{4 4}$ & $\mathbf{3 5 8}$ \\
\hline
\end{tabular}

\subsection{Comparison Results Heuristic Techniques with our Work BA, GA and the Hybrid}

This sub-section briefly explained the comparison between some previous works on heuristic algorithms. The comparison of $\mathrm{N}$-queen is standard $8 * 8$, which means the possible solution is 92 . Based on the Table 2, we compared the result with five techniques with best result achievement and applied it to hybridization of Bat algorithm with GA in order to find the best solution for N-queen. The result shows that the Bat algorithm based on the echolocation behavior of microbats is similar penalty cost with Genetic algorithms, Simulated Annealing and Randomization Method but different with another techniques.

Table 2. Comparison Results Heuristic Techniques with Bat-Algorithm

\begin{tabular}{ccl}
\hline key & $8 * 8$ & \\
\hline 1 & 0 & Bat algorithm \\
2 & 0 & Genetic algorithms Simulated Annealing and Randomization Method \\
3 & 6 & Solution of n-queen problem using ACO \\
4 & 66 & Simulated Annealing algorithm \\
5 & 1 & Genetic algorithms \\
6 & 2 & Tabu search algorithm \\
\hline
\end{tabular}

Table 3 shows the best result with three proposed techniques. The first technique was to adapt bat algorithm to solve $\mathrm{N}$-queen. The second technique was using modify GA and the last technique was a hybrid BA and GA algorithm by using two random points to split parents at this crossover point. In Table 3, the best value was obtained for the input size area of $8 * 8$ where the penalty cost is 0 similar for all three techniques. For input size area of $20 * 20$, the best value is BA-GA with penalty cost 0 , while basic bat algorithm as penalty value of 4 and the GA is 6 . For input size area of $50 * 50$ the similar penalty value was obtained from bat-GA and basic bat algorithm. While the different value for GA technique. Lastly, for input size area of $100 * 100$ and 500*500, the best results obtained was from BA-GA with best value of 26 and 272 respectively.

Table 3. Comparison Result of the Best Value with Three Different Techniques

\begin{tabular}{cccccc}
\hline Matrix size & $8 * 8$ & $20 * 20$ & $50 * 50$ & $100 * 100$ & $500 * 500$ \\
\hline BA & 0 & 4 & 8 & 48 & 330 \\
GA & 0 & 6 & 18 & 50 & 384 \\
BA-GA & 0 & 0 & 8 & 26 & 272 \\
\hline
\end{tabular}

As indicate in Figure 4, the lines colors represent the best result using three different techniques to solve $\mathrm{N}$-queen problem. The objective best solutions overall is achieved by BA-GA technique. The fig. plots the convergence run for the experimented five input size using $8 * 8,20 * 20,50 * 50,100 * 100$ and $500 * 500$. 


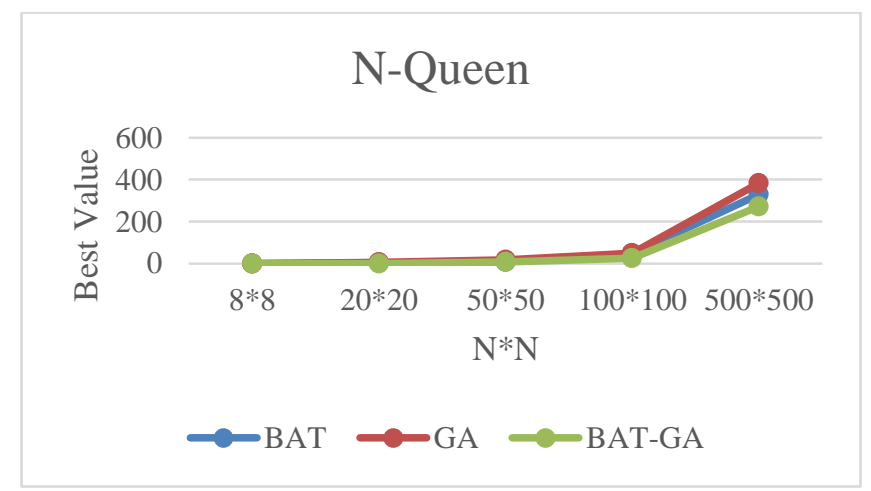

Figure 4. Convergence run for five input size area with three different techniques to get the best value for $\mathrm{N}$ Queen problems

\section{CONCLUSION}

The goal of this study is to improve the $\mathrm{N}$-queen results by adapting the bat algorithm and hybridize with GA by the adapted execution of the selection behavior in (microbats) with varying pulse rates of emissions and loudness. Two steps to evaluate all the optimal solutions that are the initialization step and moving step. Then we assess the performance on different input sizes area of $\mathrm{N} * \mathrm{~N}$ on chessboard $(8 * 8$, $20 * 20,50 * 50,100 * 100$ and $500 * 500$ ).

The result successfully shows that the hybrid algorithm is suitable to solve $\mathrm{N}$-queen problem and can be implemented on minesweeper problem as well as Sudoku puzzle problem. On further notes, this algorithm also achieved successful performance and it has potentials to solve many problems in the future because this algorithm has been applied successfully in any field, the likes of enhanced initial population for multiple protein sequence, image processing and machine learning.

\section{ACKNOWLEDGEMENTS}

Short Term Grant, Universiti Sains Malaysia, under account number 304/PKOMP/6315084, supported this work.

\section{REFERENCES}

[1] M. Bozikovic, M. Golub and L. Budin, "Solving n-queen problem using global parallel genetic algorithm," EUROCON, Ljubljana, Slovenia, 2003.

[2] I. Martinjak and M. Golub, "Comparison of heuristic algorithms for then-queen problem", Proceedings of the ITI $200729^{\text {th }}$ Int. Conf. on Information Technology Interfaces, 2007.

[3] J. Bell and B. Stevens, "A survey of known results and research areas for n-queens," Discrete Mathematics, vol. 309, pp. 1-31, 2009.

[4] S. Khan., M. Bilal., M. Sharif, M. Sajid and R. Baig, "Solution of n-queen problem using ACO," Multitopic Conference, IEEE $13^{\text {th }}$ International, 2009.

[5] A. Draa, S. Meshoul, H. Talbi, and Mohamed B, "A quantum inspired differential evolution algorithm for solving the n-queens problem," The International Arab Journal of Information Technology, Vol. 7, No. 1, 2010.

[6] F. S. Gharehchopogh, B. Seyyedi and G. Feyzipour, "a new solution for n-queens problem using blind approaches: DFS and BFS algorithms," International Journal of Computer Applications (0975 - 8887), vol. 53, pp. 45-48, 2012.

[7] B. Al-Khateeb and W. Z. Tareq. "Solving 8-queens problem by using genetic algorithms, simulated annealing, and randomization method," In Developments in eSystems Engineering (DeSE), 2013 Sixth International Conference on, pp. 187-191, 2013.

[8] I. Fister Jr., D. Fister, X. S.Yang, “A hybrid bat algorithm,” ELEKTROTEHNI'SKI VESTNIK, vol. 80, 1(2), pp. 1-7, 2013.

[9] S. Chansombat, P. Musikapun, P. Pongcharoen and C. Hicks, "A hybrid discrete bat algorithm with krill herd based advanced planning and scheduling tool for the capital goods industry," ISSN: 0020-7543 (Print) 1366-588X, 2018.

[10] J. Wang, Z. Fan, A. Zhao, and M. Yang, "A hybrid bat algorithm for process planning problem," IFACPapersOnLine, vol. 48(3), pp. 0708-1713, 2015.

[11] X. S. Yang, "a new metaheuristic bat-inspired algorithm, in: nature-inspired cooperative strategies for optimization (NISCO 2010) (Eds. J. R. Gonzalez et al.)," Studies in Computational Intelligence, Springer Berlin, vol. 284, pp. 65$74,2010$. 